\title{
Factors for Suicidal Ideation in Middle School Students by Gender
}

\author{
Seung-Kyo Chaung ${ }^{1}$. Chun-Gill Kim ${ }^{2} \cdot$ Soo Yang $^{3} \cdot$ So Young Lee ${ }^{4}$ \\ ${ }^{1}$ Department of Nursing, Semyung University, ${ }^{2}$ Division of Nursing, Hallym University, ${ }^{3}$ College of Nursing, The Catholic University of Korea, \\ ${ }^{4}$ Department of Nursing, Kkottongnae University
}

\begin{abstract}
Purpose: This study was conducted to identify the factors of suicidal ideation in middle school students by gender, and to announce the necessity of gaining upon a socio-cultural comprehension-based approach. Methods: Three middle schools were randomly selected from a pool of 21 middle schools in S City, and were stratified by school and grade. A total of 878 students were included in the analysis. Multiple logistic regression analysis was conducted to identify the factors that affect suicidal ideation by gender. Results: Suicidal ideation and depression scores of the female students were over twofold higher than those of the male students. The factor that was found to affect suicidal ideation in the male students was depression while for the female students the factors were visiting suicide-related websites, having friends who attempted to commit suicide, depression, and poor communication with one's parents. Conclusion: The findings in this study support gender differences in suicidal ideation, and suggest that building socio-cultural environments are needed to abate their negative emotions and to help youth find out their reasons to live.
\end{abstract}

Key Words: Suicidal Ideation, Depression, Comprehension, Youth

\section{INTRODUCTION}

Suicide has been reported to be the fourth highest cause of death in South Korea since 2005. In terms of age, from the teens to the thirties, suicide has been ranked as the first cause of death in both males and females. It has been the highest (29.1/100,000 persons) and was over twofold higher than the OECD average (12.1/100,000 persons) in a single year [1]. With almost one million people dying from suicide around the world each year, and with a disproportionate impact of such on the world's youth, the global society owes it to the future generations to act now with regard to such matter [2].

Suicidal ideation is distinguished from suicidal attempt and suicide but is linked to them. Although not all cases of suicidal ideation would necessarily lead to suicidal attempt or suicide, suicidal ideation has been geared to- wards suicide and could facilitate suicidal risk and the plausibility of committing suicide $[1,3,4]$. The ripple effect - that is, that suicide may impact on the lives and mental health of many families and communities, beyond the individual who commits it - dictates the need for collective action to acknowledge and address the problem as well as for commitment to effective interventions supported by political will and resources [2].

Adolescence is a transitional stage of rapid developmental change in the physical, psychological, intellectual, and social realms, which exposes one to increasing selfconsciousness and doubts about one's identity. These rapid changes can induce stressful conditions and may lead to emotional instability and impulsiveness [5-7]. According to the 2013 Korean Youth Health Behavior Survey (9th), the level of stress cognition and depression of adolescents is higher than that of adults and is higher in girls

\footnotetext{
Corresponding author: So Young Lee

Department of Nursing, Kkottongnae University, 133 Sangsam-gil, Hyeundo-myeon, Seowon-gu, Cheongju 28211, Korea.

Tel: +82-43-270-0135, Fax: +82-43-270-0614, E-mail: soyoungjh@kkot.ac.kr
}

- This study was supported by Youth Mental Health Screening Program of Siheung Mental Health Center.

Received: Nov 8, 2016 / Revised: Dec 17, 2016 / Accepted: Dec 18, 2016

This is an open access article distributed under the terms of the Creative Commons Attribution Non-Commercial License (http://creativecommons.org/licenses/ by-nc/3.0), which permits unrestricted non-commercial use, distribution, and reproduction in any medium, provided the original work is properly cited. 
than in boys and in high school students than in middle school students [8]. Suicidal ideation was also found to be higher in high school students than in middle school students, but the suicidal attempt rate was found to be higher in middle school students than in high school students [8].

Adolescents who have suicidal ideation may commit suicide in adulthood tenfold more than those who do not have suicidal ideation [7]. Also, suicidal ideation has a different elevation trend by gender, specially, suicidal ideation rate in girls has highly elevated from twelve to fourteen years old as that of boys [9]. Suicide is a concept resulting from suicidal ideation and suicidal attempt $[4,10]$, and suicidal ideation among the youth needs to be given particular attention and public health concern [9].

In previous studies, the factors that affect the adolescent suicidal ideation rate were condensed into depression, stress [4,9-12], hopelessness [2,3,14], low self-esteem [15,16], absence of social support $[2,3,5,11,15]$, bullying $[4,18]$, conflict with one's parents $[4,12,16,17]$, and poor peer relationships [4,16-18].

Besides, unlike in many other countries, the educational and social structures in South Korea have a tendency to foster crooked college admission practices and emphasize earning a degree rather than learning and ability, which has resulted not only in biased education but also in an imbalanced educational culture, particularly examinationoriented education and early childhood education. Hence, adolescents have come to have stressful conditions that could worsen their anxiety and depression [4]. Specially, family environmental stress and academic stress that affect to suicidal ideation in adolescents have been reported in Korean researches [10,17].

Legislation for suicide prevention, including fostering a life-respecting culture, has been enacted in 2011. Concurrently, Korea Suicide Prevention Center has established and implemented various relevant programs. In school, the student health promotion program has come to emphasize mental health, and this trend has expanded generally, but the scope of the program is still limited, and it is focused on preventive and general education, excepting specific strategies. To identify adolescents' mental health status and to connect which can lay down their suicidal ideation, an approach based on socio-cultural comprehension is needed. Although suicidal ideation rate was higher in high school students than in middle school students, this study focused on middle school students considering their conditional differences; elevation of academic stress in general, emotional instability, depression, and decreasing of self-esteem.

This study was conducted to identify the factors for sui- cidal ideation in middle school students by gender, and to announce the necessity of gaining upon socio-cultural comprehension based approach.

\section{METHODS}

\section{Study Design}

This study used a cross-sectional survey research design, conducting secondary analysis of screening survey data from Siheung Mental Health Center in Gyeonggi Province, South Korea. The screening survey was conducted to examine the youth's mental health status and to gather basic data for use in coming up with a community-based mental health program. The study received ethical approval from the Institutional Review Board of J Oriental Hospital, which is affiliated with S University (SMJOH, 2010-4).

\section{Setting and Sample}

This study was conducted in three middle schools randomly selected from a pool of 21 middle schools in S City. The sample was stratified by school and grade (3 classes per grade, 9 classes in one school). A total of 18 classes were selected, and a total of 906 students from the three schools agreed to participate in the study. During the data screening, the 28 participants who only partially completed the survey questionnaire were dropped out of the study. As such, a total of 878 participants were included in the analysis. Based on the power calculation using the $G^{*}$ Power program, effect size $\mathrm{f}^{2}=0.15, \alpha=.05$, power (1$\beta)=.95$, and Odds 1.5 , and considering depression the main variable related to suicidal ideation, as in the previous research [19], 417 participants were needed for the multiple logistic regression model. The actual number of participants in this study was sufficient.

\section{Procedure}

Data collection was conducted from November 1 to December 10, 2010. Three middle schools were randomly picked from a pool of 21 middle schools in S City. Three classes were randomly assigned in each grade, and nine classes per school. A document reflecting the research purpose and a request for cooperation was sent to the principal of each school, but direct contact was established with the target participants to obtain their informed consent to participate in the study. After the explanation of the research purpose to them, the students signed the informed-consent form. The ethical-consideration infor- 
mation that the students' participation was not mandatory and could be withdrawn at any time during the study was given to the students in advance.

\section{Measures}

\section{1) Suicidal ideation}

Suicidal ideation was measured using the Korean Suicidal Ideation Questionnaire (KSIQ), which is a modified version [20] of the Reynolds Suicidal Ideation Questionnaire (RSIQ) developed by Reynolds [21]. The 30 items of the RSIQ elicit data about a person's thoughts about and views of suicidal ideation. Each item is scored based on a 7-point Likert scale ranging from 0 to 6 . The means across all the items are summed up to obtain the total score (range: 0 180); a higher score indicates a higher level of suicidal ideation. The reliability was .96 for the males and .98 for the females in this study. The cut-off score for suicidal ideation was 20 , reported as having the optimal clinical utility based on the results of a clinical study involving adolescents aged 13 18[22]. In this study, the cut-off score for suicidal ideation was chosen by this clinical utility report.

\section{2) Academic stress}

Academic stress was measured using the Visual Ana$\log$ Scale (VAS), a $10 \mathrm{~cm}$ horizon bar ranging from 0 (left end) to 10 (right end). Each subject marked his/her own academic stress level on the instrument, and a higher mark represents a higher level of academic stress. Most of the other existing academic stress scales are included in general stress inventories or consist of many items, which might elevate the dropout rate or lessen the reliability of the results obtained. VAS is based on the intensity of the respondent's conformity to each of the items, and can evaluate the evaluation object easily, but its validity is still being debated [23]. Hence, by targeting 119 middle school students, partial correlation analysis was conducted between VAS and Adolescent Academic Stress Scale, which is used for the Korean Adolescent Panel Survey [17], to establish concurrent validity $(\mathrm{r}=.736, p<.001)$.

\section{3) Depression}

Depression was measured using Beck Depression Inventory II (BDI II)[24], which consists of 21 items. Each item is scored based on a 4-point Likert scale ranging from 0 (not at all) to 3 (severely). The means across all the items are summed up to obtain the total score (range: $0 \sim 63$ ); a higher score indicates a higher level of depression. The reliability was .90 in this study.

\section{4) Self-esteem}

Self-esteem was measured using VAS, which was described earlier. Each subject marked his/her own self-esteem level on the instrument; a higher mark indicates a higher level of self-esteem. Although the validity of VAS is still being debated [23], the instrument is based on the intensity of the respondent's conformity to each of the items, and can evaluate the evaluation object easily. To address VAS's weakness in terms of validity, targeting 119 middle school students, partial correlation analysis was conducted between VAS and Adolescent Self-Esteem Scale, which is used for the Korean Adolescent Panel Survey [17], to establish concurrent validity $(\mathrm{r}=.779, p<.001)$.

\section{5) Communication with one's parents}

To measure the respondent's level of communication with his/her parents, a subscale (8 items related to communication with one's parents) of Inventory of Parent and Peer Attachment (IPPA) developed by Armsden and Greenberg (1987)[25] was used. Each item is scored based on a 4-point Likert scale ranging from 1 (never) to 4 (almost every day). The means across all the items are summed up to obtain the total score (range: 8 32); a higher score indicates frequent communication with one's parents. The reliability was .78 in this study.

\section{Data Analysis}

Descriptive statistics were used to evaluate the characteristics of the subjects by gender, and the $x^{2}$ test was used to compare the differences in suicidal ideation according to the general characteristics and suicide-related variables of the subjects by gender. The independent t-test was used to compare the subjects' academic stress, depression, selfesteem, and communication with their parents according to suicidal ideation by gender. To identify the factors that affect suicidal ideation by gender, multiple logistic regression analysis was conducted. For data analysis, SPSS/WIN 16.0 was used.

\section{RESULTS}

\section{Characteristics of the Subjects and Suicidal ideation relating variables}

Except for $10.5 \%$ of the subjects, the subjects had midlow school grades (boys: $90.0 \%$; girls: $88.9 \%$ ). Most of the subjects claimed that their family income was under 2 million Korean won per month. With regard to the subjects' health behavior $39.3 \%$ of all the subjects were ad- 
dicted to the Internet, and in particular. $8.4 \%$ had friends who had attempted to commit suicide while $2.1 \%$ had family members who had attempted to do the same. And there were gender differences in suicidal ideation, academic stress, and depression. The subject female students had significantly higher scores in suicidal ideation, academic stress, and depression compared to the subject male students $(p<.001)$. Especially, the suicidal ideation in the subject female students was over twofold higher than that in the subject male students (boys: 13.3\%; girls: $31.1 \%$ )(Table 1).

\section{Suicidal Ideation according to the General Char- acteristics and Suicidal Ideation Relating Var- iables by Gender}

Among the subject male students, there were significant group differences in suicidal ideation according to whether or not they visited suicide-related websites $(p<$ .001 ) and whether or not they had friends who had attempted to commit suicide $(p=.008)$. Among the subject female students, there were also significant group differences in suicidal ideation according to whether or not

Table 1. General Characteristics and Suicidal Ideation Related Variables of the Subjects

$(N=878)$

\begin{tabular}{|c|c|c|c|c|c|}
\hline \multirow{2}{*}{ Variables } & \multirow{2}{*}{ Categories } & \multirow{2}{*}{$\frac{\text { Total }(N=878)}{\mathrm{n}(\%) \text { or } \mathrm{M} \pm \mathrm{SD}}$} & \multirow{2}{*}{$\frac{\text { Male }(n=473)}{n(\%) \text { or } M \pm S D}$} & \multirow{2}{*}{$\begin{array}{l}\text { Female }(\mathrm{n}=405) \\
\mathrm{n}(\%) \text { or } \mathrm{M} \pm \mathrm{SD}\end{array}$} & \multirow{2}{*}{$x^{2}$ or $\mathrm{t}(p)$} \\
\hline & & & & & \\
\hline \multirow[t]{3}{*}{ Grade } & 7 & $295(33.6)$ & $158(33.4)$ & $137(33.8)$ & \multirow[t]{3}{*}{$1.39(.497)$} \\
\hline & 8 & $287(32.7)$ & $153(31.3)$ & $145(34.3)$ & \\
\hline & 9 & $296(33.7)$ & $174(35.3)$ & $129(31.9)$ & \\
\hline \multirow[t]{2}{*}{ Religion } & Have & $463(52.7)$ & $232(49.0)$ & $231(57.0)$ & \multirow[t]{2}{*}{$5.59(.021)$} \\
\hline & None & $415(47.3)$ & $241(51.0)$ & $174(43.0)$ & \\
\hline \multirow[t]{3}{*}{ Academic performance } & Excellent & $92(10.5)$ & $47(9.9)$ & 45 (11.1) & \multirow[t]{3}{*}{$0.32(.851)$} \\
\hline & Moderate & $391(44.5)$ & $212(44.8)$ & $179(44.2)$ & \\
\hline & Poor & $395(45.0)$ & $214(45.2)$ & $181(44.7)$ & \\
\hline \multirow[t]{2}{*}{ Living with parents } & Yes & 748 (85.2) & 397 (83.9) & $351(86.7)$ & \multirow[t]{2}{*}{$1.29(.294)$} \\
\hline & No & $130(14.8)$ & $76(16.1)$ & $54(13.3)$ & \\
\hline \multirow{3}{*}{$\begin{array}{l}\text { Family income } \\
\text { (10,000 won/month) }\end{array}$} & $<200$ & $191(24.9)$ & 83 (19.7) & $108(31.4)$ & \multirow[t]{3}{*}{$16.33(<.001)$} \\
\hline & $200 \sim 400$ & $444(58.0)$ & $254(60.2)$ & $190(55.2)$ & \\
\hline & $>400$ & $131(17.1)$ & $85(20.1)$ & $46(13.4)$ & \\
\hline \multirow[t]{2}{*}{ Smoking } & Smoke & $158(18.0)$ & $107(22.6)$ & $51(12.6)$ & \multirow{2}{*}{$14.87(<.001)$} \\
\hline & None & $720(82.0)$ & $366(77.4)$ & $354(87.4)$ & \\
\hline \multirow[t]{2}{*}{ Alcohol consumption } & Drink & 105 (12.0) & $59(12.5)$ & $46(11.4)$ & \multirow[t]{2}{*}{$0.26(.677)$} \\
\hline & None & $773(88.0)$ & $414(87.5)$ & $359(88.6)$ & \\
\hline \multirow[t]{2}{*}{ Internet addiction } & Yes & $345(39.3)$ & $237(50.1)$ & $108(26.7)$ & \multirow[t]{2}{*}{$50.25(<.001)$} \\
\hline & No & $533(60.7)$ & $236(49.9)$ & $297(73.3)$ & \\
\hline \multirow[t]{2}{*}{ Visiting suicide-related websites } & Yes & $28(3.2)$ & $9(1.9)$ & $19(4.7)$ & \multirow[t]{2}{*}{$5.49(.021)$} \\
\hline & No & $850(96.8)$ & $464(98.1)$ & $386(95.3)$ & \\
\hline \multirow{2}{*}{$\begin{array}{l}\text { Having friends who have } \\
\text { attempted to commit suicide }\end{array}$} & Yes & $74(8.4)$ & $15(3.2)$ & $59(14.6)$ & \multirow[t]{2}{*}{$36.72(<.001)$} \\
\hline & No & $804(91.6)$ & $458(96.8)$ & $346(85.4)$ & \\
\hline \multirow{2}{*}{$\begin{array}{l}\text { Having family members who } \\
\text { have attempted to commit suicide }\end{array}$} & Yes & $18(2.1)$ & $8(1.7)$ & $10(2.5)$ & \multirow[t]{2}{*}{$0.66(.418)$} \\
\hline & No & $860(97.9)$ & $465(98.3)$ & $395(97.5)$ & \\
\hline \multirow[t]{2}{*}{ Suicidal ideation score $^{\dagger}$} & $\geq 20$ & $189(21.5)$ & $63(13.3)$ & $126(31.1)$ & $40.88(<.001)$ \\
\hline & $<20$ & $689(78.5)$ & $410(86.7)$ & 279 (68.9) & \\
\hline Suicidal ideation & $(0 \sim 180)$ & $15.59 \pm 27.05$ & $9.44 \pm 18.13$ & $22.78 \pm 33.29$ & $-7.20(<.001)$ \\
\hline Academic stress & $(0 \sim 10)$ & $6.07 \pm 2.24$ & $5.77 \pm 2.36$ & $6.43 \pm 2.05$ & $-4.41(<.001)$ \\
\hline Depression & $(0 \sim 63)$ & $10.29 \pm 8.41$ & $8.81 \pm 7.36$ & $12.02 \pm 9.21$ & $-5.66(<.001)$ \\
\hline Self-esteem & $(0 \sim 10)$ & $6.73 \pm 2.38$ & $6.84 \pm 2.44$ & $6.61 \pm 2.32$ & $1.40(.162)$ \\
\hline Communication with one's parents & $(8 \sim 32)$ & $21.50 \pm 4.94$ & $21.25 \pm 4.82$ & $21.80 \pm 5.07$ & $-1.67(.096)$ \\
\hline
\end{tabular}

${ }^{\dagger}$ Cut-off score for suicidal ideation: 20. 
Table 2. Differences in Suicidal Ideation according to the General Characteristics and Suicidal Ideation Related Variables ( $N=878)$

\begin{tabular}{|c|c|c|c|c|c|c|c|}
\hline \multirow{4}{*}{ Variables } & \multirow{4}{*}{ Categories } & \multicolumn{6}{|c|}{ Suicidal ideation $^{\dagger}$} \\
\hline & & \multicolumn{2}{|c|}{ Male $(n=473)$} & \multirow{3}{*}{$x^{2}(p)$} & \multicolumn{2}{|c|}{ Female $(n=405)$} & \multirow{3}{*}{$x^{2}(p)$} \\
\hline & & $<20$ & $\geq 20$ & & $<20$ & $\geq 20$ & \\
\hline & & $\mathrm{n}(\%)$ & $\mathrm{n}(\%)$ & & $\mathrm{n}(\%)$ & $\mathrm{n}(\%)$ & \\
\hline \multirow[t]{3}{*}{ Grade } & 7 & $132(83.5)$ & $26(16.5)$ & \multirow{3}{*}{$\begin{array}{l}2.65 \\
(.266)\end{array}$} & 87 (63.5) & $50(36.5)$ & \multirow{3}{*}{$\begin{array}{l}2.98 \\
(.226)\end{array}$} \\
\hline & 8 & $133(89.9)$ & $15(10.1)$ & & 98 (70.5) & $41(29.5)$ & \\
\hline & 9 & $145(86.8)$ & $22(13.2)$ & & 94 (72.9) & $35(27.1)$ & \\
\hline \multirow[t]{2}{*}{ Religion } & Yes & $196(84.5)$ & 36 (15.5) & \multirow{2}{*}{$\begin{array}{l}1.91 \\
(.178)\end{array}$} & $157(68.0)$ & $74(32.0)$ & \multirow{2}{*}{$\begin{array}{l}0.21 \\
(.666)\end{array}$} \\
\hline & No & $214(88.8)$ & $27(11.2)$ & & $122(70.1)$ & $52(29.9)$ & \\
\hline \multirow[t]{3}{*}{ Academic Performance } & Excellent & $42(89.4)$ & $5(10.6)$ & \multirow{3}{*}{$\begin{array}{l}0.99 \\
(.609)\end{array}$} & $32(71.1)$ & $13(28.9)$ & \multirow{3}{*}{$\begin{array}{l}0.36 \\
(.833)\end{array}$} \\
\hline & Moderate & $186(87.7)$ & $26(12.3)$ & & $125(69.8)$ & $54(30.2)$ & \\
\hline & Poor & $182(85.0)$ & $32(15.0)$ & & $122(67.4)$ & 59 (32.6) & \\
\hline \multirow[t]{2}{*}{ Living with parents } & Yes & $348(87.7)$ & 49 (12.3) & \multirow{2}{*}{$\begin{array}{l}2.04 \\
(.153)\end{array}$} & $247(70.4)$ & $104(29.6)$ & \multirow{2}{*}{$\begin{array}{l}2.69 \\
(.115)\end{array}$} \\
\hline & No & $62(81.6)$ & $14(18.4)$ & & $32(59.3)$ & $22(40.7)$ & \\
\hline \multirow{3}{*}{$\begin{array}{l}\text { Family income } \\
(10,000 \text { won/month })\end{array}$} & $<2$ & $67(80.7)$ & 16 (19.3) & \multirow{3}{*}{$\begin{array}{l}3.39 \\
(.184)\end{array}$} & $70(64.8)$ & 38 (35.2) & \multirow{3}{*}{$\begin{array}{l}1.10 \\
(.576)\end{array}$} \\
\hline & $2 \sim 4$ & $223(87.8)$ & 31 (12.2) & & $133(70.0)$ & $57(30.0)$ & \\
\hline & $>4$ & $76(89.4)$ & 9 (10.6) & & 33 (71.7) & $13(28.3)$ & \\
\hline \multirow[t]{2}{*}{ Smoking } & Smoke & $92(86.0)$ & $15(14.0)$ & \multirow{2}{*}{$\begin{array}{l}0.59 \\
(.872)\end{array}$} & $21(41.2)$ & $30(58.8)$ & \multirow{2}{*}{$\begin{array}{c}20.91 \\
(<.001)\end{array}$} \\
\hline & None & $318(86.9)$ & $48(13.1)$ & & $258(72.9)$ & $96(27.1)$ & \\
\hline \multirow[t]{2}{*}{ Alcohol consumption } & Drink & $51(86.4)$ & 8 (13.6) & \multirow{2}{*}{$\begin{array}{l}0.03 \\
(1.00)\end{array}$} & $19(41.3)$ & $27(58.7)$ & \multirow{2}{*}{$\begin{array}{l}18.42 \\
(<.001)\end{array}$} \\
\hline & None & $359(86.7)$ & $55(13.3)$ & & $260(72.4)$ & 99 (27.6) & \\
\hline \multirow[t]{2}{*}{ Internet addiction } & Yes & 209 (88.6) & 27 (11.4) & \multirow{2}{*}{$\begin{array}{l}1.44 \\
(.279)\end{array}$} & 58 (53.7) & $50(46.3)$ & \multirow{2}{*}{$\begin{array}{l}15.85 \\
(<.001)\end{array}$} \\
\hline & No & $201(84.8)$ & $36(15.2)$ & & $221(74.4)$ & $76(25.6)$ & \\
\hline Visiting suicide-related & Yes & $3(33.3)$ & $6(66.7)$ & 22.62 & $1(5.3)$ & $18(94.7)$ & 37.65 \\
\hline websites & No & 407 (87.7) & $57(12.3)$ & $(<.001)$ & $278(72.0)$ & $108(28.0)$ & $(<.001)$ \\
\hline Having friends who have & Yes & $9(60.0)$ & $6(40.0)$ & 9.55 & $23(39.0)$ & $36(61.0)$ & 28.82 \\
\hline attempted to commit suicide & No & $401(87.6)$ & $57(12.4)$ & $(.008)$ & $256(74.0)$ & $90(26.0)$ & $(<.001)$ \\
\hline Having family members who & Yes & $7(87.5)$ & $1(12.5)$ & 0.05 & $4(40.0)$ & $6(60.0)$ & 3.99 \\
\hline $\begin{array}{l}\text { have attempted to commit } \\
\text { suicide }\end{array}$ & No & $403(86.7)$ & $62(13.3)$ & $(1.00)$ & $275(69.6)$ & $120(30.4)$ & $(.077)$ \\
\hline
\end{tabular}

${ }^{\dagger}$ Cut-off score for suicidal ideation: 20.

they were smokers, whether or not they drank alcohol, and whether or not they were addicted to the Internet $(p<$ .001). That is, among both the subject male and female students, those who visited suicide-related websites and who had friends who had attempted to commit suicide were found to have significantly higher suicidal-ideation scores than those who did not visit suicide-related websites and did not have friends who had attempted to commit suicide (Table 2).

\section{Differences in Academic Stress, Depression, Self-esteem, and Communication with Parents according to Suicidal Ideation by Gender}

Based on the cut-off score for suicidal ideation (20), in both the subject male and female students, the academic stress and depression were significantly higher in the suicidal-ideation group than in the non-suicidal-ideation group while the self-esteem and communication with parents were significantly lower in the suicidal-ideation group than in the non-suicidal-ideation group, whose suicidal-ideation score was under $20(p<.001)$ (Table 3$)$.

\section{Factors affecting Suicidal Ideation by Gender}

To identify the factors that affect suicidal ideation in middle school students, multiple logistic analysis was conducted. For independent variables, significant variables both male and female students which were analyzed in descriptive analysis were included. Among suicidal ideation relating variables, visiting suicide related websites and having friends who have attempted suicide 
which were examined nominal variables changed to dummy variables.

For the subject male students, the factor that was found to affect suicidal ideation was depression; a 1 point increase in the depression score led to 1.2-fold higher odds of suicidal ideation (95\% CI: 1.13 1.26). For the subject female students, those who visited suicide-related websites had 28-fold higher suicidal-ideation scores than those who did not (95\%CI: 2.74 285.74), and those who had friends who had attempted to commit suicide got 3.40 -fold higher suicidal-ideation scores than those who did not (95\% CI: 1.57 7.38). Also, a 1 point increase in the depression score led to 1.165-fold higher odds of suicidal ideation (95\% CI: 1.12 1.22), and a 1 point increase in the communication with parents score led to 0.91 -fold lower odds of suicidal ideation (95\% CI: 0.86 0.97)(Table 4).

\section{DISCUSSION}

Social interests for suicide have been increased as the highest suicide mortality rate, and many studies on suicidal ideation have been conducted in Korea. This study was conducted not only to identify the factors for suicidal ideation in middle school students by gender, but also to announce the necessity of gaining upon socio-cultural comprehension based approach which offset suicidal ideation in middle school students.

There were differences in the suicidal-ideation scores by gender according to the general characteristics of the subjects. Jeon and colleagues [18] reported that conflicts and stress with one's peer group and conflicts with one's parents were the common predictable variables related to suicidal ideation. Conflicts with one's parents were ranked

Table 3. Differences in Academic Stress, Depression, Self-esteem, and Communication with Parents according to Suicidal Ideation

$(N=878)$

\begin{tabular}{|c|c|c|c|c|c|c|c|}
\hline \multirow{4}{*}{ Variables } & \multirow{4}{*}{ Range } & \multicolumn{6}{|c|}{ Suicidal ideation $^{\dagger}$} \\
\hline & & \multicolumn{2}{|c|}{ Male $(n=473)$} & \multirow{3}{*}{$\mathrm{t}(p)$} & \multicolumn{2}{|c|}{ Female $(n=405)$} & \multirow{3}{*}{$\mathrm{t}(p)$} \\
\hline & & $<20$ & $\geq 20$ & & $<20$ & $\geq 20$ & \\
\hline & & $\mathrm{M} \pm \mathrm{SD}$ & $\mathrm{M} \pm \mathrm{SD}$ & & $\mathrm{M} \pm \mathrm{SD}$ & $\mathrm{M} \pm \mathrm{SD}$ & \\
\hline Academic stress & $0 \sim 10$ & $5.60 \pm 2.27$ & $6.90 \pm 2.61$ & $-4.17(<.001)$ & $6.03 \pm 1.97$ & $7.31 \pm 1.95$ & $-6.07(<.001)$ \\
\hline Depression & $0 \sim 63$ & $7.30 \pm 5.83$ & $18.65 \pm 8.59$ & $-10.13(<.001)$ & $8.41 \pm 6.38$ & $20.03 \pm 9.46$ & $-12.56(<.001)$ \\
\hline Self-esteem & $0 \sim 10$ & $7.13 \pm 2.19$ & $4.91 \pm 3.01$ & $5.63(<.001)$ & $7.11 \pm 2.01$ & $5.50 \pm 2.58$ & $6.24(<.001)$ \\
\hline $\begin{array}{l}\text { Communication with } \\
\text { one's parents }\end{array}$ & $8 \sim 32$ & $21.72 \pm 4.64$ & $18.16 \pm 4.87$ & $5.63(<.001)$ & $23.01 \pm 4.44$ & $19.13 \pm 5.38$ & $7.09(<.001)$ \\
\hline
\end{tabular}

${ }^{\dagger}$ Cut-off score for suicidal ideation: 20.

Table 4. Factors Influencing Suicidal Ideation by Gender

$(N=878)$

\begin{tabular}{|c|c|c|c|c|c|c|c|c|c|c|c|}
\hline \multirow{2}{*}{ Variables } & & \multicolumn{5}{|c|}{ Male $(n=473)$} & \multicolumn{5}{|c|}{ Female $(n=405)$} \\
\hline & & $\beta$ & SE & OR & $95 \% \mathrm{CI}$ & $p$ & $\beta$ & SE & OR & $95 \% \mathrm{CI}$ & $p$ \\
\hline $\begin{array}{l}\text { Visiting suicide-related } \\
\text { websites }\end{array}$ & (1: yes; 0: no) & 1.76 & 0.95 & 5.83 & $0.90 \sim 37.77$ & .064 & 3.32 & 1.19 & 28.00 & $2.74 \sim 285.74$ & .005 \\
\hline $\begin{array}{l}\text { Having friends who } \\
\text { have attempted suicide }\end{array}$ & (1: yes; 0: no) & 0.25 & 0.94 & 1.29 & $0.20 \sim 8.17$ & .789 & 1.23 & 0.40 & 3.40 & $1.57 \sim 7.38$ & .002 \\
\hline Academic stress & & 0.00 & 0.08 & 1.00 & $0.86 \sim 1.17$ & .981 & 0.14 & 0.08 & 1.15 & $0.99 \sim 1.34$ & .071 \\
\hline Depression & & 0.18 & 0.03 & 1.19 & $1.13 \sim 1.26$ & .000 & 0.15 & 0.02 & 1.17 & $1.12 \sim 1.22$ & .000 \\
\hline Self-esteem & & -0.12 & 0.08 & 0.89 & $0.76 \sim 1.03$ & .105 & -0.05 & 0.07 & 0.95 & $0.82 \sim 1.10$ & .510 \\
\hline $\begin{array}{l}\text { Communication with } \\
\text { one's parents }\end{array}$ & & -0.06 & 0.04 & 0.94 & $0.87 \sim 1.01$ & .104 & -0.09 & 0.03 & 0.91 & $0.86 \sim 0.97$ & .004 \\
\hline Constant & & & & & -2.118 & & & & & -1.875 & \\
\hline Degree of freedom & & & & & 1 & & & & & 1 & \\
\hline
\end{tabular}


as the factor with the greatest effect in both the subject male and female students, followed by bullying and familial economic status in the subject male students and by conflicts with one's peer group and familial economic status in the subject female students. Other studies reported that familial economic status could adversely affect adolescent mental health, smoking, and alcohol consumption [11]. Also, Internet suicide site accessibility was added to the factors affecting suicidal ideation, as well as conflicts with familial members or friends [2].

Based on the cut-off score for suicidal ideation (20), the academic stress and depression were higher and the selfesteem and communication with one's parents were lower in the suicidal-ideation group than in the non-suicidalideation group. In previous studies, self-esteem $[15,16]$, familial alliance $[12,16,18]$, and social support $[2,3,5,15]$ were found to lessen the danger of suicidal ideation. In this study, self-esteem and communication with one's parents presented a similar effect of abating suicidal ideation. Depression was cited as a critical factor in suicidal ideation psychologically $[4,10-13]$, and life events preceded suicidal ideation $[2,4,14,26]$. As discussed earlier, the suicidal ideation in the subject female students in this study was twofold higher than in the subject male students, and the smoking and alcohol consumption rates were higher in the suicidal-ideation group than in the non-suicidalideation group among the subject female students. It is presumed that smoking and alcohol consumption need to be more carefully observed or monitored in female students than in male students.

Suicide impacts the most vulnerable of the world's populations, and places a larger burden on the low- and middle-income countries [2], and this economic vulnerability could be linked to other poor conditions, not only to the factors impacting suicidal ideation among adolescent $[5,11,18]$. In this study, the characteristics and environment of the subjects regarded that they were socioeconomically disadvantaged: $82.9 \%$ of the students lived in a family that their family income were under 4,000,000 won per month (family median income was 4,220,000 won per month in Korea), especially $24.9 \%$ were under 2,000,000 won; and Internet-addicted, 39.3\%; and low school grades, $45.0 \%$. These socio-economically disadvantaged conditions have resulted in adolescents' depression and suicidal ideation [12].

Also, in suicidal ideation relating variables, gender differences were found in terms of visiting suicide-related websites, having friends who have attempted to commit suicide, academic stress, and depression; more female students visited suicide-related websites and had friends who have attempted to commit suicide, and the academic stress and depression in the female students were significantly higher than those in the male students. It is not irrelevant that in previous studies, the depression level of the female students was higher than that of the male students $[4,12,14]$, and the female students could be more sensitive in terms of stress [12,26]. Phillips et al., [15] reported that negative emotions strongly associated with current and future self-harm in both male and female adolescents, while self-esteem strongly moderated current and future self-harm. It is suggested that negative emotions and response to neutral life events should be attenuated and should hold gender differentiated approach.

Shim and Kim [19] revealed that the suicidal ideation relating factors differ by phase; in early youth, stress and depression are the most powerful factors, and in mid- and late youth, the social support and impact of one's relationship with a significant key person determined the level of one's suicidal ideation. On the contrary, Kim [27] found that self-efficacy, which has a protective effect on earlyyouth suicidal ideation, decreases in mid- and late youth while the impact of depression is maintained. Another study presented participation in sports teams in and outside of school protected against suicidal ideation [13]. Considering gender and youth phase, suicidal ideation rate in boys showed indifferences, while the rate in girls showed peak elevation in age twelve to fourteen years old and relieved after age fifteen years old [9]. Although the differences depending on the youth phase and gender have not been verified, it may be appropriate to approach the clients considering the youth phase they are in for the suicidal ideation prevention program's effectiveness.

The multiple logistic regression analysis revealed that in the male students, depression was the sole factor that influenced suicidal ideation, while in the female students, visiting suicide-related websites, having friends who have attempted to commit suicide, depression, and communication with one's parents influenced suicidal ideation. Especially, among the female students, those who visited suicide-related websites showed a 28 -fold higher suicidalideation risk than those who did not, which is more than quadruple that among the male students. Although Shim and Kim [19] showed that the suicidal ideation relating factors are indifferent by gender, Jeon and colleagues [18] reported that academic performance, family economic state, conflicts with one's parents, bullying, and conflicts with one's friend related with suicidal ideation, and these are prominent in girls than those of boys. And family environmental stress and academic stress that affect to suicidal ideation in adolescents have been reported $[10,17]$. In 
this study, academic stress had related in suicidal ideation but didn't affect to suicidal ideation in logistic analysis both male and female students. Further study is needed to identify various stress inducing variables to confirm suicidal ideation affecting factors.

In Smahel and colleagues' [28] research, digital media including internet use and technology impacted physical and mental health problems to children. It is presumed that female students who have a negative mental state and strong emotional stress due to their personal conditions, including their familial environment, have a great risk to suicidal ideation. Further research should investigate whether internet addiction and visiting suicide-related website are correlated.

In the Guidelines for the Prevention of Suicide, the United Nations cites the following key components of national suicide prevention strategies: clear objectives, relevant risk and protective factors, and effective interventions. Further, the intervention targets should be divided into the general population, the vulnerable subpopulations at risk, and individuals [29]. Also, gatekeeper training, crisis care, scaling up human resources, and access to and management of persons at risk should be considered, as well as restricting access to means of inflicting self-harm and development of policies aimed at reducing the risk [2]. In South Korea, rapid growth and development may induce a work-oriented society and an inordinately education-oriented culture, particularly examination-oriented education and early childhood education. These tight and rigid atmospheres can worsen adolescents' stress; especially, they can aggravate adolescents' depression and provoke negative thinking $[4,11]$. Also, negative emotions and low self-esteem strongly associated with current and future self-harm in adolescents [15].

Not a few adolescents have faced the danger of suicide; in fact, the leading cause of death among teens at present is suicide in Korea, and the level of stress cognition and depression of adolescents is higher than that of adults [8]. To combat suicide and to prevent suicidal ideation in middle school students, we have to try to take socio-cultural comprehension based approach, and to build an environment which can abate negative emotion and draw out reason for living including making meaningful relations.

\section{Limitations}

The limitations of this study should be presented before planning the later study. First, this study was based on the respondents' self-report in response to each item. And academic stress and self-esteem were assessed through
VAS instead of based on a scale with documented reliability, although concurrent validity was applied to address VAS's weakness in validity. If researchers considered another approach in depth, such as focus group interview, we could find more diverse and copious factors which affect to negative conditions of adolescents and intimate conditions that draw in their suicidal ideation. Further research should be conducted to bring more reliable result and evidence to this concern.

\section{CONCLUSION}

Most research on suicidal ideation of adolescents in Korea has focused on analyzing risk factors, and the factors contributing to suicidal ideation by gender have reported inconsistently in each study. This study was conducted to identify the factors for suicidal ideation in middle school students by gender, and to suggest the needs for socio-cultural comprehension based approach which put down their suicidal ideation. Suicidal ideation scores of the female students were over twofold higher than those of the male students. The factor that was found to affect suicidal ideation in the male students was depression while for the female students the factors were visiting suicide-related websites, having friends who attempted to commit suicide, depression, and poor communication with one's parents.

Definitely, we should take responsibility in combating suicide by building a socio-cultural environment as well as preventing and managing the suicide risk of adolescents: diminution of negative emotion, making school culture which generate encouraging and consolation, and drawing out the reason for living. It would be possible when family, school, community including policymakers strives to develop diverse socio-cultural environment.

\section{Conflict of Interest}

The authors declared no conflict of interest.

\section{Acknowledgement}

This work was conducted secondary analysis of screening data from Siheung Mental Health Center in Gyeonggi Province, South Korea, which was pursued to examine the youth's mental health status and to gather basic data for use in coming up with a community-based mental health program. We thank the school staff who coordinated and supported the Youth's Mental Health Status Survey, and the students who participated in the research. 


\section{REFERENCES}

1. The Organization for Economic Co-operation and Development. OECD health data: Health statistics [Internet]. Paris: Author; 2014 [Cited 2015 April 15]. Available from:

http://www.oecd-ilibrary.org/social-issues-migration-health /suicides-2014-1_suicide-table-2014-1-en.

2. World Health Organization. Public health action for the prevention of suicide [Internet. Geneva: Author; 2012 [Cited 2015 April 10]. Available from:

http://www.who.int/mental_health/publications/prevention _suicide_2012/en/

3. Kuo WH, Gallo JJ, Eaton WW. Hopelessness, depression, substance disorder, and suicidality. Social Psychiatry and Psychiatric Epidemiology. 2004;39:497-501.

http://dx.doi.org/10.1007/s00127-004-0775-z

4. Wang RH, Lai HJ, Hsu HY, Hsu MT. Risk and protective factors for suicidal ideation among Taiwanese adolescents. Nursing Research. 2011;60(6):413-421.

http://dx.doi.org/10.1097/NNR.0b013e3182337d83

5. Cheng Y, Li X, Lou C, Sonenstein FL, Kalamar A, Jejeebhoy S, et al. The association between social support and mental health among vulnerable adolescents in five cities: findings from the study of the well-being of adolescents in vulnerable environments. Journal of Adolescent Health. 2014;55(6):31-38.

http://dx.doi.org/10.1016/j.jadohealth.2014.08.020

6. Langille DB, Asbridge M, Kisely S, Rasic D. Suicidal behaviours in adolescents in Nova Scotia, Canada: Protective associations with measures of social capital. Social Psychiatry Psychiatric Epidemiology. 2011;47(10):1549-1555. http://dx.doi.org/10.1007/s00127-011-0461-x

7. Reinherz HZ, Tanner JL, Berger SR, Beardslee WR, Fitzmaurice GM. Adolescent suicidal ideation as predictive of psychopathology, suicidal behaviour, and compromised functioning at age 30. The American Journal of Psychiatry. 2006;163(7):12261232. https://doi.org/10.1176/appi.ajp.163.7.1226

8. Korea Centers for Disease Control and Prevention. 2013 Korean Youth Health Behaviour Survey (9th) [Internet]. Chongwon: Author; 2013 [Cited 2015 April 16]. Available from: http://yhs.cdc.go.kr/

9. Boeninger DK, Masyn KE, Feldman BJ, Conger RD. Sex differences in developmental trends of suicide ideation, plans, and attempts among European American adolescents. Suicide and Life-Threatening Behavior. 2010;40:451-464.

http://dx.doi.org/10.1521/suli.2010.40.5.451

10. Kim BY, Lee CS. A meta-analysis of the variables related to suicidal ideation in adolescents. Journal of Korean Academy of Nursing. 2009;39(5):651-661.

http://dx.doi.org/10.4040/jkan.2009.39.5.651

11. Gong Y, Zhang L, Wang Z, Liang Y. Pathway analysis of risk factors for severe suicidal ideation: A survey in rural China. Canadian Journal of Public Health. 2011;102(6):472-475.

http://dx.doi.org/10.17269/cjph.102.2504

12. Ng LC, Kirk CM, Kanyanganzi F, Smith Fawzi MC, Sezibera V, Shema E, et al. Risk and protective factors for suicidal ideation and behaviour in Rwandan children. The British Journal of Psychiatry. 2015;207(3):262-268.

http://dx.doi.org/10.1192/bjp.bp.114.154591

13. Dugas E, Low NCP, Rodriguez D, Burrows S, Contreras G, Chation $\mathrm{M}$, et al. Early predictors of suicidal ideation in young adults. Canadian Journal of Psychiatry.2012;57(7):429-436. http://dx.doi.org/10.1177/070674371205700706

14. Stewart SM, Kennard BD, Lee PWH, Mayers T, Hughes C, Emslie G. Hopeless and suicidal ideation among adolescents in two cultures. Journal of Child Psychology and Psychiatry. 2005;46(4):364-372. http://dx.doi.org/10.1111/j.1469-7610.2004.00364

15. Phillips R, Spears M, Montgomery AA, Millings A, Sayal K, Stallared P. Could a brief assessment of negative emotions and self-esteem identify adolescents at current and future risk of self-harm in the community? A prospective cohort analysis. BioMed Central Public Health. 2013;13:604-614. http://dx.doi.org/10.1186/1471-2458-13-604

16. Lee SY. Reasons for living and their moderating effects on Korean adolescents' suicidal ideation. Death Studies. 2011;35:711728. http://dx.doi.org/10.1080/07481187.2011.553316

17. Park JY, Chung IJ. The effects of high school students' academic problems on suicidal ideation: Focusing on the mediational effects of individual-level risk and protective factors. The Journal of Korean Society of Child Welfare. 2010;32:69-97.

18. Jeon GS, Park SY, Cho S. Gender differences in correlates of depression and suicidal ideation among Korean adolescents. The Korean Journal of Health Service Management. 2012;6(4):295308. http://dx.doi.org/10.12811/kshsm.2012.6.4.295

19. Shim MY, Kim KH. Risk and protective factors for suicidal ideations of Korean adolescents: Moderating effect of sex and developmental period. The Korean Journal of Health Psychology. 2005;10(3):313-325.

20. Shin MS. An empirical study of the mechanism of suicide: Validation of the scale for escape from the self [dissertation]. Seoul: Yonsei University; 1992. p. 1-139.

21. Reynolds WM. (1988). Suicidal Ideation Questionnaire: Professional manual. Odessa FL: Psychological Assessment Resources; 1988. p. 1-50.

22. Pinto A, Whisman MA, McCoy KJM. Suicidal ideation in adolescents: Psychometric properties of the suicidal ideation questionnaire in a clinical sample. Psychological Assessment. 1997; 9(1):63-66. https://doi.org/10.1037/1040-3590.9.1.63

23. Torrance GW, Feeny D, Furlong W. Visual analog scales: Do they have a role in the measurement of preferences for health 
states?. Medical Decision Making. 2001;21:329-334. http://dx.doi.org/10.1177/0272989X0102100408

24. Beck AT, Steer RA, Ball R, Ranieri WF. Comparison of Beck Depression Inventories -IA and -II in psychiatric outpatients. Journal of Personality Assessment. 1996;67:588-597. http://dx.doi.org/10.1207/s15327752jpa6703_13

25. Armsden GC, Greenberg MT. The inventory of parent and peer attachment: Individual differences and their relationship to psychological well-being in adolescence. Journal of Youth and Adolescence. 1987;16(5):427-454. http://dx.doi.org/10.1007/BF02202939

26. Gunnell D, Harbord R, Singleton N, Jenkins R, Lewis G. Factors influencing the development and amelioration of suicidal thoughts in the general population. The British Journal of Psy- chiatry. 2004;185:385-393.

http://dx.doi.org/101192/bjp.185.5.385

27. Kim W. The effects of psychological factors in suicidal ideation among Korean youth. Studies on Korean Youth. 2014;25(3): 199-231. http://dx.doi.org/ 1014816/sky.2014.08.74.199

28. Smahel D, Wright MF, Cernikova M. The impact of digital media on health: children's perspectives. International Journal of Public Health. 2014;60:131-137.

http://dx.doi.org/10.1007/s00038-015-0649-z

29. United Nations. Prevention of suicide: Guidelines for the formulation and implementation of national strategies. New York: U.N. Department of Policy Coordination and Sustainable Development; 1996. P. 1-34. 